\title{
Analysis on Chemical Mechanization of Coal Spontaneous Combustion
}

\author{
Mingfang Xie \\ Wuhan Polytechnic, Wuhan,430000, China
}

\author{
Keywords: Coal. Spontaneous Combustion. Chemical mechanization
}

\begin{abstract}
Coal mining and processing is the main production line for coal industry, among which the importance of coal storage can not be ignored, although it seems to be easy. That is because coal would spontaneously combust when the storage reaches certain milestones. Spontaneous combustion is an important issue for storage, which needs some chemical and physical conditions. This article, from the aspect of spontaneous combustion, aims to analyze the conditions and puts emphasis on the analysis of chemical mechanization of coal spontaneous combustion. Therefore, the necessary conditions of spontaneous combustion will be concluded.
\end{abstract}

\section{Introduction}

Coal spontaneous combustion is a catastrophic phenomenon, which would cause huge losses to coal production enterprises and hinder the normal operation of enterprises. As to coal enterprises, what they should do is to try their best to prevent coal spontaneous combustion to reduce the losses. Spontaneous combustion is a phenomenon combining physic and chemistry, so there is more complex chemical mechanization in this process. If we have a clear understand of the chemical mechanization and lessen the rate of spontaneous combustion to some extent, the losses can be largely reduced. Therefore, the normal operation of enterprises can be ensured and energy waste caused by spontaneous combustion is cut down. This article, from the aspect of analysis on chemical mechanization of coal spontaneous combustion, resolves the existing questions and realizes the efficient protection from spontaneous combustion.

\section{Analysis of the causes of coal spontaneous combustion}

The production of coal is relative easier, which can be summarized and concluded in a way of assembly line. The mining is the most complex and tedious work, which effects the quality and efficiency coal to some extent. Although the storage of coal, comparing with the production processes, is not as complex as mining, its importance can not be ignored. Coal spontaneous combustion usually happens in the process of storage. Because of the external environment, the conditions of spontaneous combustion are satisfied. Therefore, spontaneous combustion occurs.

\section{Analysis of basic process of coal spontaneous combustion}

As one of the most important fuels and energies, coal is usually stored in piles, which is called coal pile. Coal pile can make oxidation reaction with oxygen to generate heat if it is stored in the outside. When a coal pile is larger, too much heat would be generated after oxidation reactions and the temperature of the pile would elevate. And the elevated temperature would once again promote the oxidation reaction to some extent, which would cause the temperature higher and higher. Finally, the temperature is high enough to reach the ignition point, spontaneous combustion is triggered.

\section{Analysis of the chemical conditions of coal spontaneous combustion}

From the above analysis, we can easily conclude that the key of coal spontaneous combustion is the oxidation reaction between coal and oxygen in the air, which would release heat to elevate the temperature to the ignition point. Analysis from another aspect is that coal spontaneous combustion is related to ventilation. Poor ventilation would cause the oxidation heat accumulation, which would be impossible to dissipate. Therefore, spontaneous combustion occurs. We can simply conclude: the spontaneous combustion expands gradually from outside to inside. Besides, the spontaneous combustion is also one of coal's chemical properties, especially for the coal of poor quality, usually 
called inferior coal, whose capacity to absorb heat from environment is relatively strong. However, from the actual analysis, what are the external factors and conditions to cause coal spontaneous combustion?

Firstly, vapor; moisture, which usually exists as vapor, is the inevitable existence in the air. It is one of the main factors to cause coal spontaneous combustion. Vapor is formed by gasification, which will absorb a lot of heat. But vapor would condensate after encountering coal, which is a heat-released reaction. The heat would be transferred to coal by vapor. From this aspect, if coal spontaneous combustion happens, we should not use water to put out the fire. Because water can not reduce the temperature, on the contrary it will elevate the temperature; as a result, the spontaneous combustion would be more intense.

Secondly, air circulation; the rate of air circulation is one of the most important factors to affect coal spontaneous combustion. In theory, the higher the rate of air circulation is; the lower probability of coal spontaneous combustion. From the aspect of chemistry, although the air circulates quickly so that the oxygen is abundant, the heat is brought out by air circulation; therefore, it is less possible for coal spontaneous combustion. If the circulation is slow, the heat would accumulate and the temperature would rise up to the ignition point. Then spontaneous combustion happens.

Thirdly, the volume of coal; the volume of coal is related to spontaneous combustion. If the coal volume is larger, even though its surface area is large, yet the covered volume is more. However, when the coal volume is small, the coal would fully contact with oxygen in the air, so it is easy for oxidation reaction and heat is generated.

Fourthly, the volatilization of coal dust; it is easy for coal to volatilize if it is piled for a long time. Therefore, much fine dust would exist in the air, whose ignition point is comparatively low. The dust would spontaneously combust, but it can not cause coal spontaneous combustion. However, it would release heat in the process of combustion, which would cause the temperature of coal pile to elevate. As the heat accumulates, coal spontaneous combustion happens.

Fifthly, the influence of temperature; temperature is the necessary condition for coal spontaneous combustion. If the temperature increases, coal would reach ignition point because the heat can not be removed in time. As a result, coal spontaneous combustion happens.

In conclusion, from the above five points, we know that some chemical environment and conditions are necessary for coal spontaneous combustion. Chemical reaction must be provided with reaction materials and conditions based on the analysis of the process of chemical reaction.

\section{Analysis of the chemical mechanization of coal spontaneous combustion}

The chemical reaction of coal spontaneous combustion can be simply summarized as oxidation reaction. That means coal spontaneous combustion is not complex, it is just oxidation reaction. However, oxidation reaction is exothermal reaction, which can cause coal spontaneous combustion. The two are complementary. So what is the real chemical mechanization for coal spontaneous combustion?

Firstly, the reasons for spontaneous combustion of coal piles are combustible substances. Coal is made from organic and inorganic by analyzing coal composition. But the basic element is carbon, and hydrogen and oxygen follow. If coal is not treated by desulfurization, sulfur must be included. All of these elements are combustive. There are certainly some incombustible substances in coal, such as ash and moisture, which are inert substances.

Secondly, there are some pyrites in coal, which is a kind of impurity. But pyrites is also one of the chemical mechanizations causing coal spontaneous combustion. For example, oxidation reaction happens to pyrites, which produces ferric oxide and sulfur anhydride. Much heat is generated in this process, which is one of the main reasons of coal spontaneous combustion. However, coal without pyrite could also spontaneously combust by statistical analysis. As a result, pyrite is not the main reason of coal spontaneous combustion, but catalytic. Therefore, coal spontaneous combustion is an oxidation reaction, in which combustive substances in coal occur oxidation reaction to some extent, and combustible is produced. Then spontaneous combustion happens. 
Finally, analyze chemical mechanization of coal spontaneous combustion. The biggest feature of chemical reaction is its process, so what are chemical process and mechanization for coal spontaneous combustion?

Firstly, water adsorption stage for coal; this stage is the initial of chemical reaction, although it belongs to physical reaction in a strict significance. Coal does not react with water in this period. But after absorbing water, coal would give out heat and the temperature of coal would increase.

Secondly, chemical adsorption stage; this stage is when chemical reaction happens and the conditions for chemical reaction is formed gradually. First is the reaction temperature, which can reach 70 degrees Celsius in general. Then oxide comes into being, resulting the phenomenon of adsorption. This is called chemical adsorption stage. At this stage, the weight of coal would increase. In addition, some gas is produced in this stage, among which carbon monoxide is the main gas. Carbon monoxide, in the process of oxidation, would make the temperature of coal increase and it is the preparation of coal spontaneous combustion.

Thirdly, complex oxidation substances of coal begin to spontaneously combust; the oxidation gases referred in the above article are all reaction substances of spontaneous combustion. The temperature of coal at this moment is very high and it has reached the ignition point. The spontaneous combustion would happen immediately. Low spontaneous combustion of coal happens in the initial stage, so the combustion rate and temperature is not very fast. However, as the aggravation of combustion, the rapid combustion stage comes. Sufficient reaction materials are necessary for the rapid combustion, because temperature has reached ignition temperature and all coal becomes reaction materials. The amount of oxygen is adequate, because coal is piled outdoors to some extent. Therefore, the chemical reaction of coal gets the greatest support, and spontaneous combustion can be completed.

Summing up the above chemical mechanization, the main is the oxidation reaction conditions. Then the combustive substances are produced, which promote the constancy of chemical reaction.

\section{Conclusion}

Coal is one of the most important energies, which plays a significant role in industry all around the world. Coal is non renewable resource for human beings, and to some extent it influences the future development of human world. Detailed analysis and scheme should be made for coal mining, production and usage. The greatest threat to coal storage is spontaneous combustion. Coal spontaneous combustion is simple physical and chemical reaction mechanization. If protective measures are well carried out, the rate of spontaneous combustion will be reduced to some extent, and the purpose of saving energy will be achieved. From the above analysis, we can sum up that the reasons for coal spontaneous combustion are vapor, ventilation, temperature and the volatilization of coal dust and so on. The main chemical reaction mechanization is oxidation reaction, which needs some conditions. As long as we can prevent the conditions of oxidation reaction, the coal spontaneous combustion will be stopped or avoided. Therefore, the analysis of chemical mechanization can reduce the rate of coal spontaneous combustion to some extent, and the purpose of saving energy can be achieved.

\section{References}

[1] Liu Dewen, Du Yuren, Wirless Prediction System of Coal Spontaneous Combustion based on Flushbonading[J], Information Technology, 2012(10):8-10

[2] Qin Yi, Yang Shengqiang, Yan Jiacheng, Du Zhenyu, Ma Xudong, Analysis and Prevention of Accidence Tree of Coal Spontaneous Combustion[J], Journal of Safety Science and Technology, 2012(8):198-204

[3] Wu Lide, Application to ShuangYang Coal Mine of Liquid Carbon Dioxide to Prevent spontaneous combustion, Heilongjiang science and technology information, 2012(6):10 